\title{
ACTIVIDAD CATALÍTICA DE METALES DE TRANSICIÓN EN LA DESCOMPOSICIÓN DE PERÓXIDO DE HIDRÓGENO
}

\author{
José G. Carriazo*, Luis. F. Bossa-Benavides y Eliana Castillo
}

Departamento de Química, Facultad de Ciencias, Universidad Nacional de Colombia, Carrera 30 No. 45-03, Bogotá - Colombia

Recebido em 1/7/11; aceito em 12/1/12; publicado na web em 11/4/12

\begin{abstract}
CATALYTIC ACTIVITY OF TRANSITION METALS IN THE HYDROGEN PEROXIDE DECOMPOSITION. This paper compares the catalytic activities of some transition metal ions $\left(\mathrm{Fe}^{3+}, \mathrm{Co}^{2+}, \mathrm{Cu}^{2+}, \mathrm{Ni}^{2+}, \mathrm{Zn}^{2+}\right)$ in the $\mathrm{H}_{2} \mathrm{O}_{2}$ decomposition in homogenous and heterogeneous processes, including solid mixed systems ( $\mathrm{Fe}-\mathrm{Cu}-\mathrm{Co} / \mathrm{Al}_{2} \mathrm{O}_{3}, \mathrm{Fe}-\mathrm{Cu} / \mathrm{Al}_{2} \mathrm{O}_{3}, \mathrm{Fe}-\mathrm{Co} / \mathrm{Al}_{2} \mathrm{O}_{3}$ and $\mathrm{Co}-\mathrm{Cu} / \mathrm{Al}_{2} \mathrm{O}_{3}$ ). The solids were characterised by X-ray diffraction to explore evolution of phases or possible changes. Different trends of the catalytic activity were observed: in homogeneous medium the most active species was $\mathrm{Fe}^{3+}$, whereas in heterogeneous one the higher activities were shown for $\mathrm{Co} / \mathrm{Al}_{2} \mathrm{O}_{3}$ and $\mathrm{Co}-\mathrm{Cu} / \mathrm{Al}_{2} \mathrm{O}_{3}$. A strong cooperative effect for the $\mathrm{Co}-\mathrm{Cu} / \mathrm{Al}_{2} \mathrm{O}_{3}$ system was observed, which can be considered as a new catalyst of interest for this type of reactions.
\end{abstract}

Keywords: hydrogen peroxide; transition metals; catalysis by metals.

\section{INTRODUCCIÓN}

Hoy en día, la búsqueda de nuevos catalizadores para la degradación (oxidación) de contaminantes orgánicos en medio acuoso impone la necesidad de explorar la actividad catalítica de nuevas especies, capaces de mejorar los niveles de oxidación y alcanzar los requerimientos ambientales impuestos por la legislación mundial. Recientemente, el peróxido de hidrógeno ha recibido atención creciente como un agente oxidante de gran interés para la eliminación de moléculas orgánicas en medio acuoso, debido a su potencial de oxido-reducción y a que los productos de su descomposición son totalmente inocuos (oxígeno y agua). ${ }^{1,2}$ Desde esta perspectiva, el empleo de especies de metales de transición, en forma de cationes, óxidos u óxidos soportados, constituye un campo de investigación que ofrece posibles soluciones en el diseño de nuevos catalizadores para la oxidación de compuestos orgánicos en medio acuoso, entre ellos el fenol, uno de los contaminantes ambientales peligrosos y frecuentes en aguas residuales de industrias, altamente tóxico y de carácter cancerígeno. ${ }^{3-8}$

En la actualidad se ha venido trabajando, en diversas partes del mundo, en el desarrollo de sistemas catalíticos para la degradación de contaminantes en medio acuoso, hasta lograr la implantación o soporte de metales de transición en la estructura molecular de sólidos naturales o sintéticos, ${ }^{6,8-10}$ con el objeto de consolidar catalizadores heterogéneos eficientes, selectivos a la formación de $\mathrm{CO}_{2}$ como producto final de la oxidación de los contaminantes orgánicos, y que faciliten su separación mediante pasos elementales en una potencial aplicación a escala industrial. ${ }^{2,11}$ Con el empleo de especies de $\mathrm{Fe}^{3+}$ y $\mathrm{Cu}^{2+}$, soportados en minerales de arcilla y otros soportes catalíticos, en la reacción de oxidación total de compuestos orgánicos en medio acuoso, se ha evidenciado la mayor actividad del hierro en presencia de peróxido de hidrógeno como molécula oxidante, pero una mayor selectividad del cobre hacia la formación de $\mathrm{CO}_{2}$ frente al mismo oxidante. ${ }^{12,13}$ Otros autores indican buena actividad catalítica para otras especies metálicas (por ejemplo, $\mathrm{Pt}, \mathrm{Ru}, \mathrm{Co}, \mathrm{Mn}, \mathrm{Cr}, \mathrm{Ni}$ ) en la misma reacción, ya sea como constituyentes de estructuras sólidas o como iones en solución; ;,4,6,10,14 sin embargo, no es claro un esquema comparativo de la actividad de diversos metales en estos

*e-mail: jcarriazog@unal.edu.co sistemas de oxidación con peróxido de hidrógeno toda vez que, su actividad catalítica es fuertemente influenciada por la naturaleza de las especies metálicas (potencial de reducción), el pH y por el soporte catalítico empleado, entre otros. ${ }^{1,15,16}$ De tal manera que, es necesario esclarecer las ventajas de algunos metales frente a otros en la conformación de sistemas catalíticos de oxidación asistida por peróxido de hidrógeno, para poder diseñar y preparar futuros catalizadores en estado sólido con mayor eficiencia y selectividad en la reacción de oxidación total de compuestos orgánicos contaminantes en medio acuoso:

$$
\mathrm{C}_{x} \mathrm{H}_{y_{(a c)}}+\mathrm{H}_{2} \mathrm{O}_{2(a c)} \stackrel{\text { Catalizador (Metal) }}{\longrightarrow} \mathrm{CO}_{2(\mathrm{~g})}+\mathrm{H}_{2} \mathrm{O}_{(l)}
$$

El estudio de metales de transición en los procesos de oxidación catalítica es fundamental para el desarrollo de nuevos catalizadores con mayor eficiencia en dichos procesos. En la actualidad se emplean los llamados procesos avanzados de oxidación (PAO), basados en la capacidad oxidativa del peróxido de hidrógeno en presencia de iones de metales de transición como $\mathrm{Fe}^{3+}$ y $\mathrm{Cu}^{2+}$, entre otros. ${ }^{6,7,17,18}$ En este contexto, el proceso Fenton $\left(\mathrm{Fe}^{2+}-\mathrm{Fe}^{3+}\right) / \mathrm{H}_{2} \mathrm{O}_{2}$, y en especial el proceso foto-Fenton $\left(\left(\mathrm{Fe}^{2+}-\mathrm{Fe}^{3+}\right) / \mathrm{H}_{2} \mathrm{O}_{2} / \mathrm{UV}\right)$, han generado gran interés en los últimos años debido a su eficiencia para degradar una gran cantidad de contaminantes orgánicos. ${ }^{6,8,17-19}$

Por otra parte, el diseño de catalizadores sólidos requiere la selección de la fase activa (especie metálica) y el soporte adecuados. Por tanto, establecer una comparación que permita clasificar diversas especies metálicas según su actividad catalítica sería un aporte deseable que permitiría establecer mejor el criterio de selección de los metales para posibles sistemas catalíticos mixtos en estado sólido aplicables a los PAO, con buenas propiedades de actividad y selectividad.

En los PAO, el peróxido de hidrógeno es descompuesto por la especie metálica, a través de la formación de radicales libres hidroxilo y perhidroxilo $\left(\cdot \mathrm{OH}\right.$ y $\left.\bullet \mathrm{O}_{2} \mathrm{H}\right)$, los cuales atacan a la molécula orgánica hasta oxidarla. ${ }^{6,7,20}$ Entonces, es posible evaluar preliminarmente la actividad de los metales empleados como catalizadores a través de la descomposición del peróxido de hidrógeno, cuyos radicales libres (en ausencia de las especies orgánicas a oxidar) finalmente forman $\mathrm{O}_{2}$ y agua: ${ }^{20-24}$

$$
\mathrm{H}_{2} \mathrm{O}_{2(a c)} \stackrel{\text { Catalizador (Metal) }}{\longrightarrow} \mathrm{O}_{2(\mathrm{~g})}+\mathrm{H}_{2} \mathrm{O}_{(\mathrm{l})}
$$


Vale la pena mencionar que, en algunos casos la descomposición de peróxido de hidrógeno puede desarrollarse por vía no radicalaria. ${ }^{20,25,26}$ Por ejemplo, bajo ciertas condiciones (soluciones alcalinas) el $\mathrm{H}_{2} \mathrm{O}_{2}$ puede disociarse formando las especies iónicas $\mathrm{H}^{+}$y $\mathrm{HO}_{2}{ }^{-}$(el anión perhidroxilo puede actuar como nucleófilo), ${ }^{20}$ y en algunas ocasiones se puede producir oxígeno y agua sin la formación de radicales hidroxilo y perhidroxilo como intermediarios (es el caso de reacciones que involucran enzimas y algunas especies inorgánicas de manganeso). ${ }^{25}$ Sin embargo, los diferentes estudios reportados en literatura indican que los iones de metales empleados en el presente trabajo (a valores de $\mathrm{pH}$ ácidos o cercanos a la neutralidad) tienden a descomponer, en mayor o menor medida, el $\mathrm{H}_{2} \mathrm{O}_{2}$ mediante la formación de radicales libres. Por tanto, en la presente investigación se trabajó bajo la hipótesis de que la descomposición del peróxido de hidrógeno se desarrolla mayoritariamente a través de la formación de radicales hidroxilo y perhidroxilo. Este aspecto se considera importante porque la velocidad de formación de estos radicales es determinante para la oxidación de compuestos orgánicos contaminantes en medio acuoso, ${ }^{25,26}$ aunque finalmente los catalizadores deben ser ensayados directamente en soluciones que contengan las moléculas orgánicas a oxidar, toda vez que algunos compuestos (como los aromáticos clorados) muestran mayor dificultad que otros para ser oxidados totalmente. De hecho, en estudios previos la oxidación catalítica de fenol en medio acuoso con peróxido de hidrógeno ha sido correlacionada cuantitativamente con la generación de radicales hidroxilo. ${ }^{26,27}$

El objetivo del presente trabajo es establecer un esquema organizado de las actividades catalíticas de especies metálicas de Fe, $\mathrm{Cu}, \mathrm{Co}, \mathrm{Ni}$ y $\mathrm{Zn}$ en la reacción de descomposición de peróxido de hidrógeno, en concentración moderadamente diluida (1\%), para las especies metálicas en medio homogéneo (sales solubles) y en medio heterogéneo (sólido insoluble). Los resultados permitirán extrapolar los sistemas catalíticamente más eficientes para su posterior optimización y aplicación en la reacción de oxidación total (eliminación) de moléculas orgánicas contaminantes mediante el uso de sistemas heterogéneos tipo Fenton $\left(\right.$ metal $/ \mathrm{H}_{2} \mathrm{O}_{2}$ ) y avanzar hacia el diseño de catalizadores altamente eficientes y estables con menor impacto sobre el equilibrio ambiental. En todo caso, los catalizadores deberán ser ensayados frente a la oxidación (con peróxido de hidrógeno) de moléculas orgánicas "modelo" en una segunda etapa de este proyecto.

\section{PARTE EXPERIMENTAL}

En cuanto a la preparación de los catalizadores, se tuvieron en cuenta tres criterios para la selección de los metales: iones de metales de transición que por su configuración electrónica pudieran alojar electrones en sus orbitales "d" (esto excluiría al Zn", sin embargo se incluyó teniendo en cuenta que es capaz de coordinarse con algunas especies ricas en oxígeno); cationes de elementos de transición considerados como NO tóxicos en concentraciones bajas (esto excluye a $\mathrm{Cr}, \mathrm{Mn}, \mathrm{Cd}, \mathrm{Hg}$, entre otros); sales de metales de muy bajo costo (esto excluye a los metales nobles) y de uso frecuente en Química.

\section{Materiales de partida}

Se emplearon sales inorgánicas (tipo reactivo analítico) de los correspondientes metales a estudiar: $\mathrm{Fe}\left(\mathrm{NO}_{3}\right)_{3} \cdot 9 \mathrm{H}_{2} \mathrm{O}$ (Merck, 99.5\%), $\mathrm{Co}\left(\mathrm{NO}_{3}\right)_{2} \cdot 6 \mathrm{H}_{2} \mathrm{O}$ (Panreac, 99.9\%), $\mathrm{NiCl}_{2} \cdot 6 \mathrm{H}_{2} \mathrm{O}$ (May \& Baker, $97 \%$ ), $\mathrm{Cu}\left(\mathrm{NO}_{3}\right)_{2} \cdot 3 \mathrm{H}_{2} \mathrm{O}$ (Panreac, $99.0 \%$ ) y $\mathrm{Zn}\left(\mathrm{NO}_{3}\right)_{2} \cdot 6 \mathrm{H}_{2} \mathrm{O}(95 \%)$. Se usó peróxido de hidrógeno Panreac al $30 \%$. Como soporte de los catalizadores heterogéneos se empleó una alúmina comercial (Sigma-Aldrich, área superficial: $155 \mathrm{~m}^{2} / \mathrm{g}$ ).

\section{Catalizadores homogéneos}

Los catalizadores homogéneos se prepararon mediante la disolución de las sales de los metales $\mathrm{Fe}, \mathrm{Cu}, \mathrm{Co}$, Ni y Zn, para alcanzar una concentración final de $0.01 \mathrm{M}$. Para la preparación de las soluciones de las sales se usó agua destilada y luego desionizada (resistividad de 14.2 M $\Omega . c m$ ). Para los ensayos catalíticos se emplearon soluciones frescas, mantenidas en recipientes de color ámbar y en un medio refrigerado para evitar su descomposición.

\section{Catalizadores heterogéneos}

Los catalizadores sólidos se prepararon mediante la impregnación húmeda de las sales metálicas $\left(\mathrm{Fe}^{3+}, \mathrm{Cu}^{2+}, \mathrm{Co}^{2+}, \mathrm{Ni}^{2+}\right.$ y $\left.\mathrm{Zn}^{2+}\right)$ sobre alúmina comercial: se adicionó $10 \mathrm{~mL}$ de solución acuosa $0.01 \mathrm{M}$ de cada ión metálico a una masa cercana a $10 \mathrm{~g}$ de $\mathrm{Al}_{2} \mathrm{O}_{3}$ (y se sometió a agitación constante por media hora) con el objeto de obtener una concentración de 0.01 milimoles de metal por gramo de catalizador. Los sólidos se secaron a $60{ }^{\circ} \mathrm{C}$ toda una noche y se calcinaron a $500{ }^{\circ} \mathrm{C}$ durante $2 \mathrm{~h}$, luego de elevar la temperatura con rampa de calentamiento a $1.4{ }^{\circ} \mathrm{C} / \mathrm{min}$, en atmósfera estática usando una mufla Thermolyne 48000 (Modelo F48015).

Por otra parte, se prepararon (por impregnación húmeda) sistemas sólidos mixtos en composición metálica ( $\mathrm{Fe}-\mathrm{Cu}-\mathrm{Co}, \mathrm{Fe}-\mathrm{Cu}, \mathrm{Fe}-\mathrm{Co}$ y $\mathrm{Co}-\mathrm{Cu}$ ), usando relaciones equimolares de los iones metálicos, con el objeto de obtener concentraciones de 0.01 milimoles totales de metal por cada gramo de catalizador. En el caso del sistema $\mathrm{Fe}-\mathrm{Cu}-\mathrm{Co}$ se emplearon 0.033 milimoles de cada metal; para Fe-Cu, Fe-Co y Co-Cu se usaron 0.050 milimoles de cada metal para $10 \mathrm{~g}$ de catalizador.

\section{Caracterización de los sólidos}

Tanto el soporte de partida (alúmina comercial y alúmina calcinada a $500{ }^{\circ} \mathrm{C}$ ) como los catalizadores sólidos se caracterizaron por difracción de rayos X (radiación $\mathrm{K} \alpha$ de $\mathrm{Cu}=1.54060 \AA$ ̊), en un equipo X-Pert Pro MPD PANalitical con geometría $2 \theta$ y configuración Bragg-Brentano, a temperatura ambiente, empleando un tamaño de paso de $0.01 \mathrm{y}$ un tiempo de paso de $10 \mathrm{~s}$.

\section{Evaluación catalítica}

La actividad catalítica se evaluó en la reacción de descomposición de peróxido de hidrógeno, en un reactor tipo batch de 250 $\mathrm{mL}$ de capacidad, totalmente cerrado, cubierto con aluminio para evitar la acción de la luz, termostatado a $25^{\circ} \mathrm{C}$ y acoplado a un potenciómetro (Hanna Instruments $\mathrm{pH} 211$ ) para el registro continuo de los valores de $\mathrm{pH}$ durante la reacción. Para cada ensayo el reactor fue cargado con $100 \mathrm{~mL}$ de solución al $1 \%$ de $\mathrm{H}_{2} \mathrm{O}_{2}$ recién preparada y sometido a agitación magnética (340 rpm), como se muestra en la Figura 1.

La reacción inicia cuando se adiciona el catalizador $(1 \mathrm{~mL}$ de solución $0.01 \mathrm{M}$ de cada metal para la reacción en medio homogéneo, ó $1 \mathrm{~g}$ de catalizador sólido para la reacción en medio heterogéneo) y el reactor se cierra rápidamente. El seguimiento de la reacción se realizó midiendo de manera continua la evolución del oxígeno formado, el cual se determinó con un sensor diferencial de de presión (referencia MPX 2010), asumiendo que el aumento de presión dentro del sistema se debe exclusivamente al oxígeno producido por la descomposición catalizada de peróxido de hidrógeno. El sensor se conectó a un multímetro (UT60E RS232C) y un sistema computarizado (software UT60E Interface Program_ver 2.02), el cual registra los datos de presión generados en unidades de milivoltios. Los datos obtenidos en milivoltios fueron convertidos a unidades de 


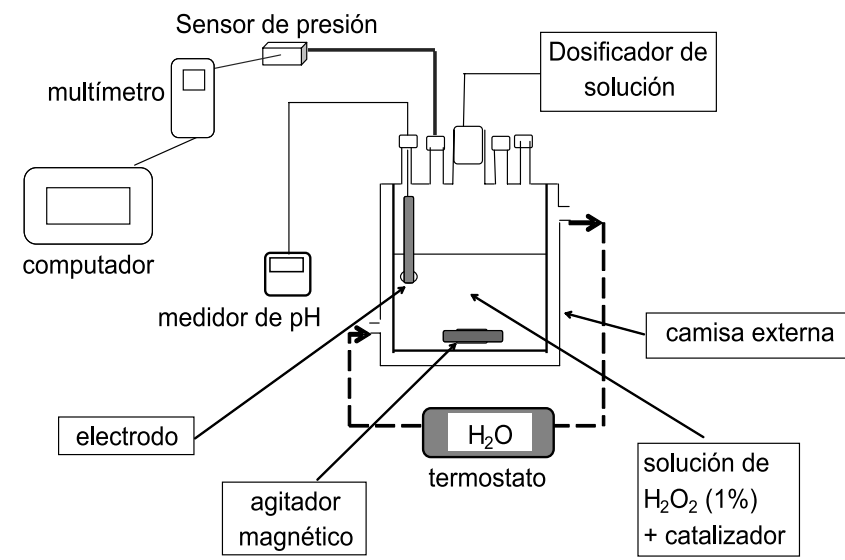

Figura 1. Esquema del reactor usado para la descomposición de peróxido de hidrógeno

presión $(\mathrm{kPa})$ y a moles de oxígeno según las siguientes ecuaciones obtenidas experimentalmente: ${ }^{28}$

$$
\frac{m V_{r e g}-0.2449 m V}{2.45 m V / K P a}=P_{r e g}(K P a)
$$

donde $\mathrm{mV}_{\text {reg }}$ es la señal de presión del sistema en milivoltios registrados desde el sensor y $\mathrm{P}_{\text {reg }}(\mathrm{kPa})$, es la presión del sistema en kilopascales.

$$
\eta_{O_{2(g a s)}}=\frac{\left|P_{r e g} v\right|}{R \cdot T}+\left[k_{h} \cdot v_{(s o l)} \cdot P_{r e g}\right]
$$

donde $\eta_{\mathrm{O}_{2(\text { gas })}}$ es el número de moles producidas, $\mathrm{R}$ es la contante de los gases, $T$ es la temperatura, $v$ es el volumen del reactor ocupado por la fase gaseosa, $k_{h}$ es la constante de Henry para la disolución de oxígeno en agua a la temperatura dada $\mathrm{y}_{(\mathrm{sol})}$ es el volumen de solución.

Todos los ensayos catalíticos se realizaron por triplicado, con error menor al $3 \%$. Aunque se conoce que la descomposición de $\mathrm{H}_{2} \mathrm{O}_{2}$ por metales es más eficiente a valores de $\mathrm{pH}$ ácidos, ${ }^{20}$ las reacciones se desarrollaron a pH espontáneo con el fin de registrar la tendencia natural de cada sistema catalítico sin forzar el equilibrio mediante controles externos de esta variable. Esto permite registrar y comparar la actividad de los sistemas catalíticos sin la adición de ácido, que en últimas podría ocasionar lixiviación metálica. ${ }^{12}$ Finalmente, al terminar cada ensayo en fase heterogénea, las soluciones se filtraron (filtro Millipore de $0.45 \mu \mathrm{m}$ ) para separar el sólido de la fase acuosa y valorar la posible lixiviación de los metales durante la reacción, mediante absorción atómica en un equipo Perkin-Elmer Analyst 300 y usando lámparas de cátodo hueco.

\section{RESULTADOS Y DISCUSIÓN}

\section{Difracción de rayos X (DRX)}

Los difractogramas obtenidos, tanto para el soporte (alúmina cruda (comercial) y alúmina calcinada) como para los catalizadores sólidos (sistemas simples y mixtos) (Figura 2), muestran las señales características de la fase $\delta$-alúmina, la cual según se indica en literatura es estable hasta temperaturas cercanas a $1000{ }^{\circ} \mathrm{C} .{ }^{29-31}$ Teniendo en cuenta que la calcinación de todos los sólidos se hizo a $500{ }^{\circ} \mathrm{C}$, este resultado muestra que el soporte es térmicamente estable y que durante la preparación de los sólidos no ocurrieron cambios estructurales (apreciables por esta técnica) sobre el mismo.
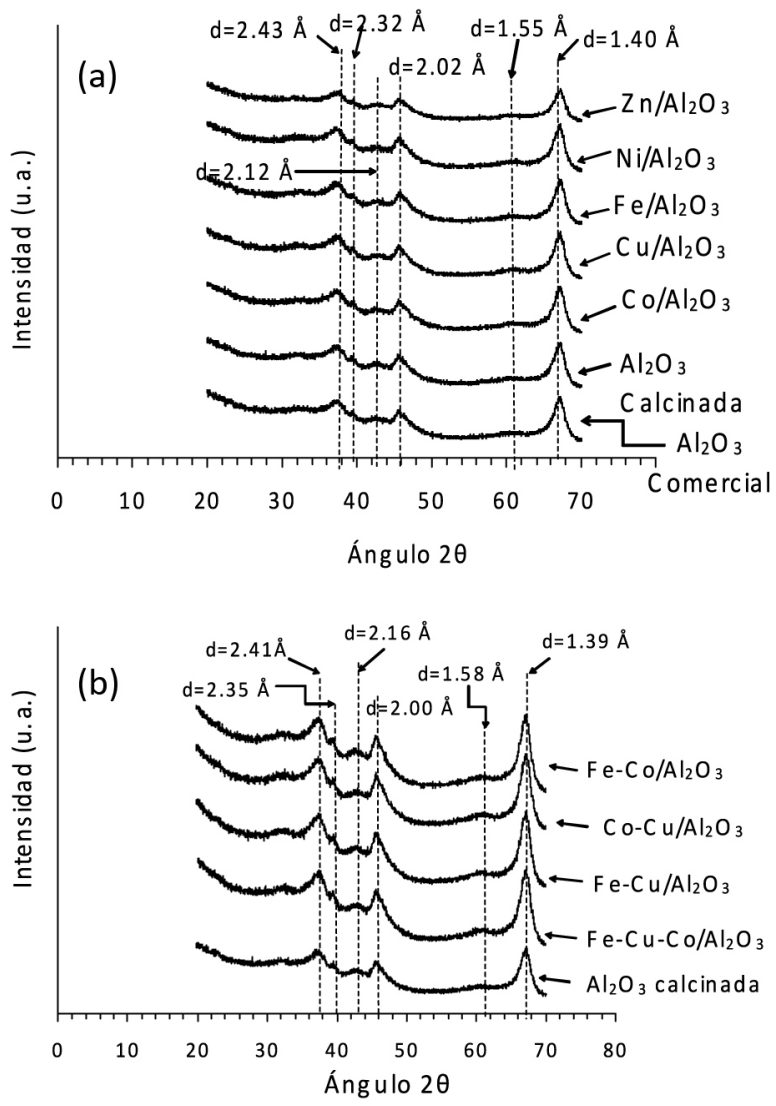

Figura 2. Difractogramas de rayos $X$ de: a) el soporte (alúmina) y de los sólidos simples ( $\mathrm{Fe}_{\mathrm{Al}} \mathrm{O}_{2} \mathrm{O}_{3}, \mathrm{Cu} / \mathrm{Al}_{2} \mathrm{O}_{3}, \mathrm{Co}_{\mathrm{Al}} \mathrm{O}_{2}, \mathrm{Ni}_{\mathrm{Al}} \mathrm{O}_{2} \mathrm{O}_{3}$ y $\mathrm{Zn} / \mathrm{Al}_{2} \mathrm{O}_{3}$ ), b) los catalizadores mixtos ( $\mathrm{Fe}-\mathrm{Co} / \mathrm{Al}_{2} \mathrm{O}_{3}, \mathrm{Co}-\mathrm{Cu} / \mathrm{Al}_{2} \mathrm{O}_{3}, \mathrm{Fe}-\mathrm{Cu} / \mathrm{Al}_{2} \mathrm{O}_{3}$ y $\mathrm{Fe}-\mathrm{Cu}-\mathrm{Co} /$ $\mathrm{Al}_{2} \mathrm{O}_{3}$ )

De otro lado, en ningún caso el análisis por difracción de rayos $\mathrm{X}$ muestra la presencia de fases metálicas o de óxidos metálicos correspondientes a los elementos que se impregnan. Es obvio que, estequiométricamente se forman óxidos de los metales impregnados cuando los sólidos se calcinan en presencia de aire (atmósfera estática), pero en el presente trabajo las cantidades de metal soportado son muy bajas ( 0.01 milimoles de metal por gramo de catalizador) y probablemente por debajo del límite de detección de la técnica, o las fases de óxidos formadas son amorfas y por tanto no detectables por DRX. Adicionalmente, no se observaron desplazamientos de los parámetros de celda (longitud de las aristas) para el soporte, luego de la impregnación y calcinación de los metales, lo cual descarta una posible incorporación de los metales mediante sustitución isomórfica en la estructura de la alúmina.

\section{Actividad catalítica en medio homogéneo}

La Figura 3 muestra los resultados de actividad catalítica de los cationes metálicos estudiados en medio homogéneo. Se observa claramente que los sistemas de mayor actividad $\left(\mathrm{Fe}^{3+}\right.$ y $\mathrm{Cu}^{2+}$, con 12.6 y $10.5 \%$ de conversión de $\mathrm{H}_{2} \mathrm{O}_{2}$ respectivamente) (mayores niveles de formación de oxígeno) corresponden a los reportados en literatura para la reacción Fenton $\left(\mathrm{Fe}^{3+} / \mathrm{H}_{2} \mathrm{O}_{2}\right)$ o tipo Fenton $\left(\mathrm{Cu}^{2+} /\right.$ $\mathrm{H}_{2} \mathrm{O}_{2}$ ). ${ }^{14}$ Los valores de $\mathrm{pH}$ registrados para cada reacción (a lo largo de cada ensayo) se indican en la Tabla 1, donde se pone en evidencia la tendencia de los sistemas catalíticos en medio homogéneo hacia los valores óptimos de $\mathrm{pH}$, indicados en literatura. ${ }^{15,16,27,32,33}$ Durante cada ensayo, el $\mathrm{pH}$ se mantuvo constante en un intervalo muy estrecho de valores: 3.5 en promedio para el $\mathrm{Fe}^{3+}$ y 5.5 en promedio para $\mathrm{el} \mathrm{Cu}^{2+}$. 


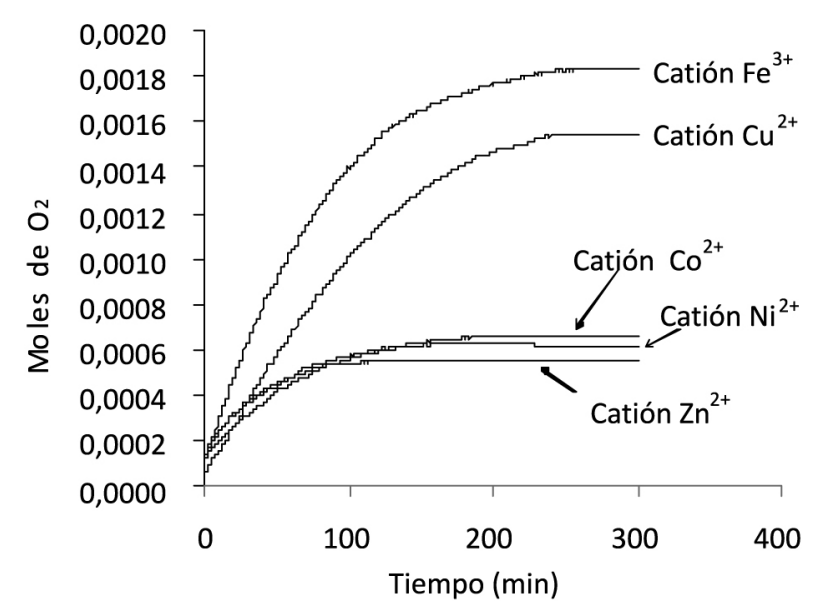

Figura 3. Actividad catalítica en la descomposición de peróxido de hidrógeno de los metales de transición en medio homogéneo $\left(\mathrm{Fe}^{3+}, \mathrm{Cu}^{2+}, \mathrm{Co}^{2+}, \mathrm{Ni}^{2+} y \mathrm{Zn}^{2+}\right)$

Tabla 1. Valores de $\mathrm{pH}$ registrados para las reacciones en medio homogéneo

\begin{tabular}{ccc}
\hline Catión & $\begin{array}{c}\text { pH promedio } \\
(3 \text { ensayos })\end{array}$ & $\begin{array}{c}\text { pH óptimo } \\
\text { reportado }\end{array}$ \\
\hline Ninguno & $3.5^{*}$ & - \\
$\mathrm{Fe}^{3+}$ & 3.5 & $3.5(\mathrm{a}, \mathrm{b})$ \\
$\mathrm{Cu}^{2+}$ & 5.5 & $5.5(\mathrm{~b})$ \\
$\mathrm{Co}^{2+}$ & 4.5 & $5.0(\mathrm{c})$ \\
$\mathrm{Ni}^{2+}$ & 4.7 & $>4.3(\mathrm{~d})$ \\
$\mathrm{Zn}^{2+}$ & 4.6 & - \\
\hline
\end{tabular}

*Valor de pH para la solución de $\mathrm{H}_{2} \mathrm{O}_{2}$ al 1\%. (a) ref. 27; (b) ref. 32; (c) ref. 16 ; (d) ref. 15

Los cationes $\mathrm{Co}^{2+}, \mathrm{Ni}^{2+}$ y $\mathrm{Zn}^{2+}$ presentaron la menor actividad (menores niveles de formación de oxígeno), comparable entre ellos pero muy baja en comparación con la actividad catalítica de las especies de hierro o de cobre. En todos los casos, las curvas de actividad catalítica (Figura 3) alcanzan la meseta (máximo de actividad, donde la pendiente es cero), mostrando que el catalizador ha alcanzado su capacidad máxima. En ninguno de los casos se observó precipitación de óxidos u oxihidróxidos a partir de los cationes metálicos en solución, lo que en principio descarta el detrimento en la actividad catalítica de $\mathrm{Ni}^{2+}$ y $\mathrm{Zn}^{2+}$ como consecuencia de la precipitación de la fase activa.

\section{Actividad catalítica en medio heterogéneo}

Para los catalizadores sólidos, los comportamientos de los distintos sistemas simples se muestran en la Figura 4a, en donde se observa claramente que la actividad catalítica de los sólidos de cobalto y de cobre (23 y $17 \%$ de conversión de $\mathrm{H}_{2} \mathrm{O}_{2}$, respectivamente) es mayor que la actividad correspondiente al catión más activo en medio homogéneo $\left(\mathrm{Fe}^{3+}\right.$, con $\left.12.6 \%\right)$. Se debe tener en cuenta que el soporte usado es la alúmina, lo cual le confiere al catalizador preparado ciertas propiedades químicas que influyen en las condiciones del medio de reacción, como el valor de $\mathrm{pH}$. Los valores de $\mathrm{pH}$ registrados fueron cercanos al valor generado en el ensayo con el soporte (alúmina como catalizador), el cual fue de 6.9. En todos los casos el pH se mantuvo dentro del intervalo de 6.3 a 7.2 (Tabla 2), lo que indica que el pH del medio de reacción es auto-controlado por la superficie del soporte.

La Figura 4a, muestra la máxima actividad para el sistema heterogéneo a base de cobalto $\left(\mathrm{Co} / \mathrm{Al}_{2} \mathrm{O}_{3}\right)$, seguido de los sistemas de
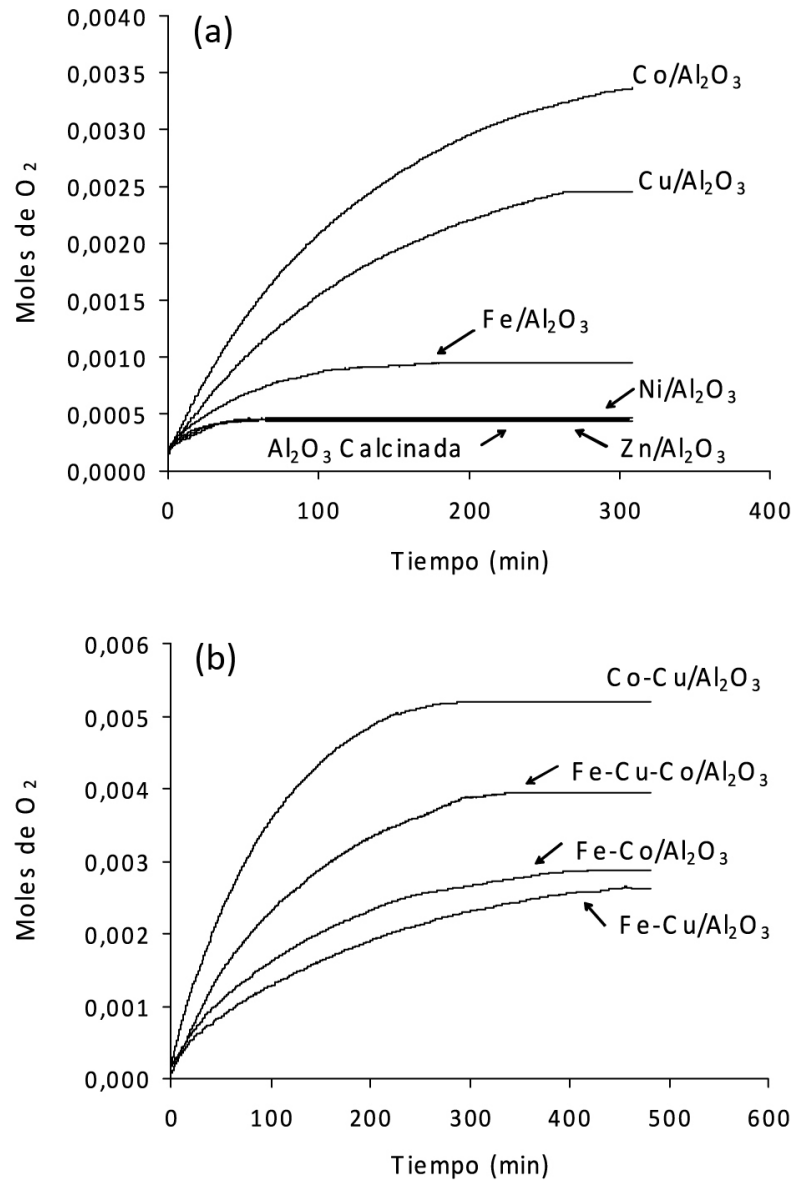

Figura 4. Actividad catalítica, en la descomposición de peróxido de hidrógeno, de los sólidos: a) simples ( $\mathrm{Fe}_{\mathrm{Al}} \mathrm{O}_{2}, \mathrm{Cu} / \mathrm{Al}_{2} \mathrm{O}_{3}, \mathrm{Co} / \mathrm{Al}_{2} \mathrm{O}_{3}, \mathrm{Ni}_{\mathrm{Al}} \mathrm{O}_{2} \mathrm{O}_{3}, \mathrm{Zn} /$ $\mathrm{Al}_{2} \mathrm{O}_{3}$ y $\mathrm{Al}_{2} \mathrm{O}_{3}$ calcinada), b) mixtos ( $\mathrm{Co}-\mathrm{Cu} / \mathrm{Al}_{2} \mathrm{O}_{3}, \mathrm{Fe}-\mathrm{Cu}$ - $\mathrm{Co} / \mathrm{Al}_{2} \mathrm{O}_{3}, \mathrm{Fe}-\mathrm{Co} /$ $\mathrm{Al}_{2} \mathrm{O}_{3}$ y $\mathrm{Fe}$ - $\mathrm{Cu} / \mathrm{Al}_{2} \mathrm{O}_{3}$ )

Tabla 2. Valores de $\mathrm{pH}$ y metal lixiviado durante las reacciones en medio heterogéneo

\begin{tabular}{ccc}
\hline Catalizador & $\begin{array}{c}\mathrm{pH} \text { promedio } \\
(3 \text { ensayos })\end{array}$ & Metal/Lixiviado \\
\hline Ninguno & $3.5^{*}$ & - \\
$\mathrm{Al}_{2} \mathrm{O}_{3}$ calcinada & 6.9 & $\mathrm{ND}$ \\
$\mathrm{Fe} / \mathrm{Al}_{2} \mathrm{O}_{3}$ & 6.6 & $\mathrm{ND}$ \\
$\mathrm{Cu} / \mathrm{Al}_{2} \mathrm{O}_{3}$ & 6.4 & $\mathrm{ND}$ \\
$\mathrm{Co} / \mathrm{Al}_{2} \mathrm{O}_{3}$ & 6.6 & $\mathrm{ND}$ \\
$\mathrm{Ni} / \mathrm{Al}_{2} \mathrm{O}_{3}$ & 6.4 & $\mathrm{ND}$ \\
$\mathrm{Zn} / \mathrm{Al}_{2} \mathrm{O}_{3}$ & 6.8 & $\mathrm{ND}$ \\
\hline
\end{tabular}

*Valor de $\mathrm{pH}$ para la solución de $\mathrm{H}_{2} \mathrm{O}_{2}$ al $1 \%$. ND: No detectado

cobre y de hierro $\left(\mathrm{Cu} / \mathrm{Al}_{2} \mathrm{O}_{3}\right.$ y $\mathrm{Fe} / \mathrm{Al}_{2} \mathrm{O}_{3}$, respectivamente). La variación en las capacidades máximas de actividad catalítica para estos sistemas heterogéneos frente a sus respectivos cationes en medio homogéneo probablemente es el resultado de una fuerte influencia del $\mathrm{pH}$, controlado por el soporte. De esta manera, es posible que los sistemas catalíticos de cobalto y de cobre, en estado sólido, se sitúen en un valor favorable de $\mathrm{pH}$, el cual desfavorece la actividad catalítica del hierro. Los sólidos a base de níquel y cinc presentan la menor actividad catalítica, con el mismo nivel de actividad de la alúmina calcinada (soporte), lo que indica que estas especies metálicas tienen una actividad nula, y que la poca actividad es proporcionada por el soporte. 
Por otra parte, la elevada actividad catalítica de los sistemas sólidos de cobalto y de cobre a valores de $\mathrm{pH}$ cercanos a la neutralidad hace que estos sólidos puedan considerarse sistemas catalíticos importantes y promisorios para futuras aplicaciones en la degradación, con peróxido de hidrógeno, de compuestos orgánicos en aguas residuales, ya que se elimina la necesidad de ajustar el $\mathrm{pH}$ a la neutralidad (frecuentemente desde valores muy ácidos) como requisito ambiental para aguas de vertimiento. Adicionalmente, mediante análisis por absorción atómica, al final de cada reacción, no se encontró metal lixiviado en ningún caso (Tabla 2), lo que indica que cualquier posible lixiviación bajo las condiciones de reacción estaría por debajo del límite de detección de la técnica. Este resultado constituye un aspecto favorable para futuras aplicaciones de estos sistemas catalíticos, toda vez que indica que éstos no generan contaminación adicional apreciable en el medio de reacción, y puede estar relacionado con las condiciones extremadamente suaves de reacción ( $\mathrm{pH}$ muy cercano a la neutralidad y temperatura ambiente) y con los contenidos de metal (fase activa), muy bajos, empleados en la preparación de los catalizadores.

\section{Actividad catalítica de los sistemas mixtos}

La Figura 4b muestra las actividades catalíticas de los sistemas mixtos empleados en la descomposición del peróxido de hidrógeno ( $\mathrm{Fe}-\mathrm{Cu}-\mathrm{Co} / \mathrm{Al}_{2} \mathrm{O}_{3}, \mathrm{Fe}-\mathrm{Cu} / \mathrm{Al}_{2} \mathrm{O}_{3}, \mathrm{Fe}-\mathrm{Co} / \mathrm{Al}_{2} \mathrm{O}_{3}$ y $\mathrm{Co}-\mathrm{Cu} / \mathrm{Al}_{2} \mathrm{O}_{3}$ ). $\mathrm{La}$ mayor actividad catalítica la presentaron los sistemas $\mathrm{Co}-\mathrm{Cu}$ y $\mathrm{Fe}$ $\mathrm{Cu}-\mathrm{Co}$, con los niveles más altos en la producción de oxígeno $(0.0053$ y 0.0039 moles de oxígeno, correspondientes al 36.1 y $26.5 \%$ de conversión de $\mathrm{H}_{2} \mathrm{O}_{2}$, respectivamente). Teniendo en cuenta que, los sólidos mixtos fueron preparados a partir de cantidades equimolares de los metales empleados como fase activa en cada caso, se podría esperar una contribución aditiva de las especies metálicas (posiblemente óxidos metálicos) en la actividad catalítica; sin embargo, en todos los casos los niveles de oxígeno obtenidos empleando sistemas mixtos son mayores que los valores esperados mediante un efecto simplemente aditivo.

La razón $(\mathrm{CR} / \mathrm{CE})$ entre la cantidad real de oxígeno obtenido $(\mathrm{CR})$ y la cantidad de oxígeno esperado por la adición de los metales (CE) fue de $1.4,1.5,1.7$ y 1.8 para los sistemas $\mathrm{Fe}-\mathrm{Co}, \mathrm{Fe}-\mathrm{Cu}, \mathrm{Fe}-\mathrm{Cu}-\mathrm{Co}$ y $\mathrm{Co}-\mathrm{Cu}$, respectivamente. Estas relaciones, bastante mayores que la unidad, muestran el efecto cooperativo en la actividad catalítica de los sistemas mixtos debido a la mezcla de las especies metálicas, e indican que dicho efecto es mayor en los sistemas $\mathrm{Fe}-\mathrm{Cu}-\mathrm{Co}$ y $\mathrm{Co}-\mathrm{Cu}$. El sólido $\mathrm{Co}-\mathrm{Cu} / \mathrm{Al}_{2} \mathrm{O}_{3}$ constituye el sistema catalítico más importante, que muestra los mayores niveles de formación de oxígeno y por tanto la mayor eficiencia en la descomposición de $\mathrm{H}_{2} \mathrm{O}_{2}$

Los valores de $\mathrm{pH}$ registrados durante la reacción, para los sistemas mixtos, estuvieron alrededor de $6.8\left(\mathrm{Co}-\mathrm{Cu} / \mathrm{Al}_{2} \mathrm{O}_{3} \mathrm{pH}=6.9 ; \mathrm{Fe}-\right.$ $\mathrm{Cu}-\mathrm{Co} / \mathrm{Al}_{2} \mathrm{O}_{3} \mathrm{pH}=7.0 ; \mathrm{Fe}-\mathrm{Co} / \mathrm{Al}_{2} \mathrm{O}_{3} \mathrm{pH}=6.9 ; \mathrm{Fe}-\mathrm{Cu} / \mathrm{Al}_{2} \mathrm{O}_{3} \mathrm{pH}=6.8$ ). De igual manera, en los sistemas mixtos se considera predominante las propiedades superficiales del soporte (superficie de la alúmina) en la tendencia y autocontrol del $\mathrm{pH}$ de la reacción. Los sistemas catalíticos mixtos, al igual que los sistemas heterogéneos simples, no mostraron lixiviación metálica detectable por absorción atómica. Este resultado es importante, puesto que posibilita la aplicación de estos sólidos como catalizadores en reacciones de oxidación con peróxido de hidrógeno en medio acuoso, sin que ocurra contaminación adicional por disolución de los metales en el medio de reacción. Los catalizadores más activos de los sistemas mixtos fueron evaluados en operaciones de reuso (Figura 1S, material suplementario), mostrando una pequeña reducción en su actividad catalítica ( $\sin$ transformación estructural apreciable por difracción de rayos X), algo común en catálisis heterogénea y que puede estar ligado a la transformación o a lixiviaciones mínimas de la fase activa (especie metálica), ya que los contenidos de metal son extremadamente bajos.

\section{CONCLUSIONES}

Los resultados del presente trabajo permiten comparar la actividad de algunos metales de transición ( $\mathrm{Fe}, \mathrm{Co}, \mathrm{Cu}, \mathrm{Ni}, \mathrm{Zn}$ ), tanto en medio homogéneo como en medio heterogéneo, en la reacción de descomposición del $\mathrm{H}_{2} \mathrm{O}_{2}$, y por tanto permiten seleccionar los sistemas catalíticos más importantes para posibles aplicaciones en reacciones de oxidación en medio acuoso empleando peróxido de hidrógeno. Los catalizadores sólidos se prepararon con mínima cantidad de cationes metálicos sobre $\mathrm{Al}_{2} \mathrm{O}_{3}$, cuya fase se identificó como $\delta$-alúmina mediante difracción de rayos $\mathrm{X}(\mathrm{DRX})$. Adicionalmente, los análisis por DRX no evidenciaron incorporación metálica por substitución isomórfica en la estructura de la alúmina, y tampoco permitieron identificar los óxidos metálicos formados sobre el soporte. Los cationes $\mathrm{Fe}^{3+}$ y $\mathrm{Cu}^{2+}$ constituyen los catalizadores homogéneos más activos, mientras que los sólidos $\mathrm{Cu} / \mathrm{Al}_{2} \mathrm{O}_{3}$ y $\mathrm{Co} / \mathrm{Al}_{2} \mathrm{O}_{3}$ son los catalizadores heterogéneos simples más importantes y los sistemas mixtos $\mathrm{Fe}-\mathrm{Cu}-\mathrm{Co} / \mathrm{Al}_{2} \mathrm{O}_{3}$ y $\mathrm{Co}-\mathrm{Cu} / \mathrm{Al}_{2} \mathrm{O}_{3}$ son los sólidos de máxima actividad catalítica. Las diferentes especies metálicas heterogéneas (simples o mixtas) fueron estables en el medio de reacción, mostrando niveles de disolución de metales no detectables por absorción atómica. Finalmente, se encontró un fuerte efecto cooperativo en las propiedades catalíticas de las especies metálicas de $\mathrm{Co}-\mathrm{Cu}$ y de $\mathrm{Fe}-\mathrm{Cu}-\mathrm{Co}$. Los valores de $\mathrm{pH}$ del medio de reacción, cercanos a la neutralidad, la elevada estabilidad de las especies metálicas en solución acuosa y la mayor actividad catalítica de estos sólidos, los convierten en sistemas importantes con grandes potenciales de aplicación en reacciones de oxidación de impacto ambiental.

\section{MATERIAL SUPLEMENTARIO}

El material suplementario contiene las gráficas de actividad catalítica para ensayos de reutilización de los dos catalizadores mixtos más importantes, y los difractogramas de rayos $\mathrm{X}$ de dichos sólidos después de ser reutilizados. Este material se encuentra disponible, en archivo PDF, con acceso libre en la página web http://quimicanova. sbq.org.br.

\section{AGRADECIMIENTOS}

Los autores agradecen a la Universidad Nacional de Colombia por el apoyo logístico.

\section{REFERENCIAS}

1. Salem, I. A.; Monatsh. Chem. 2000, 131, 1139.

2. Carriazo, J. G.; Moreno-Forero, M.; Molina, R. A.; Moreno, S.; Appl. Clay Sci. 2010, 50, 401.

3. Bhargava, S. K.; Tardio, J.; Prasad, J.; Foger, K.; Akolekar, D. B.; Gocott, S. C.; Ind. Eng. Chem. Res. 2006, 45, 1221.

4. Busca, G.; Berardinelli, S.; Resini, C.; Arrighi, L.; J. Hazard. Mater. 2008, 160, 265.

5. Suárez-Ojeda, M. E.; Carrera, J.; Metcalfe, I. S.; Font, J.; Chem. Eng. J. 2008, 144, 205.

6. Liotta, L. F.; Gruttadauria, M.; Di Carlo, G.; Perrini, G.; Librando, V.; J. Hazard. Mater. 2009, 162, 588 .

7. Iurascu, B.; Siminiceanu, I.; Vione, D.; Vicente, M. A.; Gil, A.; Water Res. 2009, 43, 1313.

8. Garrido-Ramirez, E. G.; Theng, B. K. G.; Mora, M. L.; Appl. Clay Sci. 2010, 47, 182. 
9. Salem, I. A.; El-Maazawi, M. S.; Chemosphere 2000, 41, 1173.

10. Gupta, K. C.; Sutar, A. K.; J. Mol. Catal. A 2007, 272, 64.

11. Sanabria, N. R.; Ávila, P.; Yates, M.; Rasmussen, S. B.; Molina, R.; Moreno, S.; Appl. Clay Sci. 2010, 47, 283.

12. Yip, A. C. K.; Lam, F. L. Y.; Hu, X.; Chem. Eng. Sci. 2007, 62, 5150.

13. Ñungo-Moreno, J.; Carriazo, J. G.; Molina, R.; Moreno, S.; Revista de la Academia Colombiana de Ciencias Exactas, Físicas y Naturales 2011, $35,293$.

14. Ciesla, P.; Kocot, P.; Mytych, P., Stasicka, Z.; J. Mol. Catal. A 2004, 224, 17.

15. Salem, I. A.; Elhag, R. I.; Khalil, K. M. S.; Transition Met. Chem. 2000, $25,260$.

16. El-sousy, K.; Hussen, A.; Hartani, K.; El-Aila, H.; Jordan J. Chem. 2007, 2, 97 .

17. Nogueira, R. F. P.; Trovó, A. G.; da Silva, M. R. A.; Villa, R. D.; de Oliveira, M. C.; Quim. Nova 2007, 30, 400

18. Aguiar, A.; Ferraz, A.; Contreras, D.; Rodríguez, J.; Quim. Nova 2007, 30,623 .

19. Andreozzi, R.; Caprio, V.; Insola, A.; Marotta, R.; Catal. Today 1999, $53,213$.

20. Jones, C. W.; Applications of hydrogen peroxide and derivatives, The Royal Society of Chemistry: Cambridge, 1999.
21. Gemeay, A. H.; Colloids Surf., A 1996, 116, 277.

22. Gemeay, A. H.; Salem, M. A.; Salem, I. A.; Colloids Surf., A 1996, 117, 245.

23. Pérez-Benito, J. F.; J. Phys. Chem. A 2004, 108, 4853.

24. Pérez-Benito, J. F.; J. Inorg. Biochem. 2004, 98, 430.

25. Kwan, W. P.; Voelker, B. M.; Environ. Sci. Technol. 2003, 37, 1150.

26. Huang, C. P.; Huang, Y. H.; Appl. Catal., A 2008, 346, 140.

27. Tatibouet, J. M.; Guelou, E.; Fournier, J.; Top. Catal. 2005, 33, 225.

28. Paternina-Villareal, E. J.; Tesis de Maestría, Universidad Nacional de Colombia, Colombia, 2007.

29. Lippens, B. C.; De Boer, J. H.; Acta Crystallogr. 1964, 17, 1312.

30. Lippens, B. C.; Steggerda, J. J. In Physical and chemical aspects of adsorbents and catalysis; Linsen, B. G.; Fortuin, J. M. H.; Okkerse, C.; Steggerda, J. J., eds.; Academic Press: London, 1970, p. 171-208.

31. Geiculescu, A. C.; Strange, T. F.; Thin Solid Films 2003, 426, 160.

32. Barrault, J.; Tatibouet, J. M.; Papayannakos, N.; C. R. Acad. Sci. Paris, Série IIc, Chimie/Chemistry 2000, 3, 777.

33. Carriazo, J. G.; Guelou, E.; Tatibouët, J. M.; Moreno, S.; Appl. Clay Sci. 2003, 22, 304. 